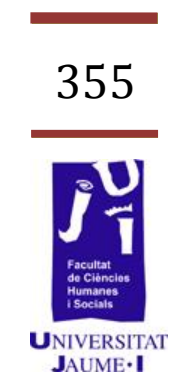

\title{
Hacia prácticas inclusivas en el CEIP Francesc Roca i Alcaide
}

Paula Escobedo Peiro pescobed@uji.es Ma Auxiliadora Sales Ciges asales@uji.es Joan A. Traver Martí jtraver@uji.es 
La presente comunicación parte de un proyecto de tesis doctoral que estudia la realidad de un centro educativo en su proceso de transformación hacia el modelo intercultural inclusivo. El problema de investigación viene determinado por la necesidad de llevar los cambios de este modelo a la realidad de las aulas de la escuela. Con el objetivo de favorecer la transformación de prácticas de enseñanza tradicionales en prácticas inclusivas. En dicho estudio, nos centramos en el seminario de formación que realizó el profesorado del centro y en las prácticas de aula observadas para conocer como se fueron transformando las prácticas docentes en el centro. Obteniendo como resultados la incorporación de prácticas de innovación educativa en gran parte de las aulas de la escuela. Como conclusiones, destacamos que el replanteamiento sobre las prácticas de aula en algunos casos promovió cambios en las concepciones pedagógicas de algunos docentes.

Palabras clave: modelo intercultural - inclusivo, comunidades de aprendizaje, investigación - acción colaborativa, participación de las familias, colaboración docente, prácticas inclusivas.

\section{El CEIP Francesc Roca i Alcaide}

Actualmente, diversas escuelas se ven en la necesidad de transformarse para poder responder a la necesidad del alumnado. Partimos de una sociedad global y diversa donde entendemos que una enseñanza homogeneizadora queda muy lejana de la realidad social que vivimos. En algunas ocasiones, la cultura escolar y la cultura de las familias que forman parte de la comunidad educativa se definen desde mundos totalmente alejados. Esta situación facilita la desconexión del alumnado de aquello escolar y la poca funcionalidad y relación entre los aprendizajes realizados en la escuela y los aprendizajes para la vida. En estas circunstancias, cada vez más escuelas se plantean la necesidad de transformarse para crear una realidad de todas y todos sus miembros.

En la presente comunicación, que parte de un proyecto de tesis doctoral, definimos algunos de los cambios realizados en una escuela que se ha planteado la transformación hacia un modelo intercultural inclusivo que responda a las necesidades del momento actual.

Para entender mejor la realidad de la escuela definimos brevemente su contexto. EI CEIP Francesc Roca i Alcaide es un CAES (Centro de Acción Educativa Singular) situado en las afueras de la localidad de Borriana (Castellón), en el barrio "La Bosca". Este barrio está mal visto en la 
localidad y en consecuencia, lo está también la escuela. Se trata de una zona repleta de viviendas de protección oficial, habitadas mayoritariamente por personas del norte de África o familias con un nivel socio-económico bajo. En esta parte de la ciudad podemos encontrar la Oficina de Servicios Sociales, la Oficina de Promoción de la Autonomía y Atención a la Dependencia y el servicio de Atención al Desarrollo Infantil (ADI). Sin embargo, no hay zonas de ocio o centros culturales. Para poder ir a la biblioteca municipal los niños han de desplazarse al centro de la localidad y difícilmente lo hacen. Según el profesorado del centro procedente de Burriana, las familias salen muy poco del barrio.

Las principales problemáticas que presenta la escuela son tres: el bajo rendimiento del alumnado, la poca y/o mala relación con las familias y la mala reputación de la escuela en la localidad, a causa del alumnado que acoge y del lugar dónde está situada. El bajo rendimiento del alumnado mayoritariamente se debe al desconocimiento de la lengua. Puesto que un $90 \%$ del alumnado habla el árabe y la lengua vehicular de la escuela es el valenciano principalmente y el castellano. La relación con las familias se explica por varias razones. Entre el profesorado y las familias árabes más que mala relación hay muy poca comunicación. Les resulta muy dificil entenderse debido al desconocimiento de las madres árabes del idioma. En el caso del $10 \%$ de las familias del barrio de origen español, por norma general, la relación no es adecuada. Antes de iniciar la transformación de la escuela algunas familias denunciaron en varias ocasiones a la escuela. En definitiva, cuando se decide iniciar el cambio en la escuela, tanto por parte de las familias hacia el profesorado como a la inversa, hay mucha desconfianza y prejuicios. Por último, la imagen negativa que se tiene de la escuela en la localidad es un aspecto que preocupa principalmente al profesorado y que se debe al lugar donde la escuela está situada. Por esta razón iniciar un proyecto que implica tanto a las familias como a vecinos del barrio pensámos que puede ser una buena iniciativa para mejorar la situación del barrio y de las personas que viven allí.

Con la prioridad de dar una respuesta adecuada a la diversidad y ofrecer una educación de calidad, el CEIP Francesc Roca i Alcaide se plantea modificar su estructura como escuela e ir incorporando elementos que fomenten una participación democrática de toda la comunidad. Su objetivo es que el alumnado aumente el rendimiento y se mejore el clima de convivencia. Aunque, generalmente, la convivencia entre el profesorado y el alumnado no supone un problema en esta escuela, encuentran dificultades tanto en la relación con algunas familias 
como en la implicación de éstas en la escuela. Por estas razones, la comunidad educativa del CEIP Francesc Roca i Alcaide a principios del curso 2011 - 2012, deciden crear una escula de todas y todos; empiezan a construir una "comunidad de aprendizaje" (CREA $\left.{ }^{1}\right)$. El profesorado, el alumnado y las familias sueñan juntos la escuela que les gustaría tener.

De todas las transformaciones que empiezan a realizarse en la escuela, nos centramos en las prácticas de aula. Como dice Booth (2006) para poder hablar de inclusión tendremos que llevar los valores de la inclusión a la acción. Para que el alumnado pueda empaparse de esos valores necesita experimentarlos desde la práctica. De ahí nuestro interés en las prácticas docentes como espacio donde adquirir los valores de la inclusión, en escuelas que así lo pretenden.

En el modelo tradicional de escuela, el profesorado asume la exclusiva del conocimiento que enseña. Comunidades de aprendizaje rompe con esta idea a través de la participación de todos los miembros de la comunidad educativa en la transformación de la escuela y de la sociedad, concretada en el conocimiento que se enseña en el aula, el modelo didáctico que se utiliza, en la base epistemológica, etc. La práctica educativa que se lleva a cabo (...) ha sido consensuada por todas las personas que integran la comunidad educativa, mediante la aplicación de una metodología comunicativa (Gómez, 2004: 423).

Por tanto, el aula es un espacio donde se concretan y afloran cuestiones que están emergiendo en el cambio del centro, donde se realizan prácticas que evidencian los valores de la inclusión y donde se construyen contextos compartidos entre la escuela y la comunidad. Sin embargo, aunque parece obvio que los cambios del centro también deberían reflejarse en las aulas, no siempre es así (Escobedo, 2010), sino que algunos elementos de la cultura escolar dificultan que los cambios lleguen a todos los rincones de la escuela (Bolívar, 1996; Ortiz y Lobato, 2003). Por eso, nuestro interés es conocer cómo y por qué se transforman las prácticas de aula, para pasar de prácticas tradicionales a prácticas inclusivas como són los grupos interactivos y las tertulias dialógicas. Por acciones educativas inclusivas entendemos «aquellas que proporcionan el apoyo necesario a todo el alumnado, manteniendo al mismo tiempo un entorno de aprendizaje común y reorganizando los recursos disponibles» (Grañeras, Díaz y Gil, 2011: 56).

\footnotetext{
${ }^{1}$ Se siguen las fases del CREA (Centro Especial de Investigación en Teorías y Prácticas Superadoras de Desigualdades http://creaub.info/es/).
} 


\section{Prácticas inclusivas: seminario de formación}

El cambio en el tipo de prácticas, además de ser un objetivo del proyecto de tesis era un sueño para la comunidad educativa. Querían realizar prácticas que favorecieran la equidad entre las personas, que aumentaran el rendimiento y mejoraran el clima de convivencia. Entre las prácticas a realizar, el modelo de comunidades de aprendizaje define los grupos interactivos y las tertulias diálogicas como acciones educativas de éxito. Por el contrario, una comunidad de aprendizaje no puede definirse como tal si no realiza estas acciones educativas de éxito.

En el mes de febrero del 2012 las maestras de primer ciclo empiezan a realizar acciones educativas de éxito con la participación de las familias. Sin embargo, gran parte del profesorado en abril del 2012 todavía no ha incorporado ningún cambio y realizan prácticas tradicionales alejadas de la inclusión. Esta situación no es extraña, sino que ya en la literatura se apunta la falta de herramientas del profesorado para dar respuesta a la diversidad (Essomba, 2006). Por eso, el profesorado de esta escuela pide formación para conocer las técnicas que pueden aplicar en su aula y conseguir mejorar el rendimiento del alumnado.

En estas circunstancias, con la necesidad de formación que se explicita entre una parte del profesorado y con la experiencia de las personas que están innovando en sus aulas, deciden realizar un seminario de formación. El seminario nace con la intención de fomentar la implementación de prácticas inclusivas.

Dicho seminario se constituye como un espacio de reflexión para la acción. Partimos de la investigación - acción colaborativa como herramienta metodológica para fomentar los cambios en las aulas del CEIP Francesc Roca i Alcaide. La investigación- acción colaborativa hace referencia a una serie de estrategias realizadas para mejorar el contexto educativo y social en el que se desarrolla (Latorre, 2003). Para Lewin (1946) la investigación, la acción y la formación son tres elementos esenciales para el desarrollo profesional. La formación del profesorado necesita de la observación sobre la acción y la indagación para resolver las problemáticas que surgen en nuestra práctica. Latorre (2003, p.24) define la investigación - acción como "una indagación práctica realizada por el profesorado, de forma colaborativa, con la finalidad de mejorar su práctica educativa a través de ciclos de acción y reflexión".

El seminario de investigación - acción se inicia a finales de abril del 2012 y finaliza a finales de junio de ese mismo año. Durante ese tiempo se realizan cinco sesiones de formación y acciones consecuentes con la 
formación recibida. La primera y la segunda sesiones las impartieron algunas maestras de educación infantil y explicaron en qué consisten los Proyectos de Trabajo. Dicha práctica no está acuñada en las comunidades de aprendizaje como acción educativa de éxito. Sin embargo, las maestras de la escuela que realizan estas prácticas manifiestan que atiende a la diversidad del aula y se alcanzan buenos resultados con el alumnado. Por eso, ellas quieren seguir realizándolos. La pretensión en los Proyectos de Trabajo es que el alumnado sea el protagonista de su aprendizaje. Para ello, el alumnado mantiene un rol activo y reflexivo en su proceso de aprendizaje y en la construcción del proyecto. Tienen que razonar y ser capaces de construir su propio aprendizaje.

En la primera sesión, las maestras explicaron las fases de los proyectos de trabajo y cómo ellas los realizaban. Desde el primer momento el resto del profesorado participó, preguntó las dudas que le iban surgiendo y manifestaron sus miedos e inseguridades. Tras dicha sesión el profesorado pidió que la segunda reunión también se destinara a Proyectos de Trabajo. En esa sesión, el profesorado estuvo preguntando dudas para conocer cómo podría realizar un proyecto en su clase. Acordaron llegar a compromisos mínimos, para que las prácticas explicadas llegaran a la acción. Sin embargo, estas dos sesiones de reflexión y formación no alcanzaron su acción hasta a principios del siguiente curso. Para el que se acordó realizar al menos un Proyecto de Trabajo a lo largo del curso.

La tercera sesión la prepararon las maestras de primer ciclo. Ellas, desde el inicio de la transformación empezaron a introducir nuevas técnicas en sus aulas. Como hemos comentado anteriormente, introdujeron los grupos interactivos y las tertulias dialógicas. Pero lo más interesante de su experiencia fueron los buenos resultados que se obtuvieron con la participación de algunas familias en sus aulas.

A la sesión de formación que ellas prepararon invitaron también a las familias que entraban en sus clases para que contaran como vivían la experiencia. Al final sólo pudo asistir una madre a la sesión de formación y valoró la experiencia como muy positiva y enriquecedora. Además, la madre insistió en la limitación que supone para ellas el desconocimiento del idioma. Aunque las maestras insistieron en que la entrada de estas madres en el aula no suponía ninguna dificultad, sino que posibilitaba el aprendizaje de la lengua también para ellas.

Los aspectos destacados en la sesión de formación sobre la experiencia fueron la alta motivación del alumnado, la mejora en el clima de convivencia del aula y los distintos aprendizajes que se adquirieron mediante estas técnicas y que no hubieran podido alcanzar trabajando 
como lo hacían anteriormente. Como por ejemplo, la cooperación entre el alumnado, el fomento de la autonomía, etc. También destacaron algunos aspectos negativos como la rigidez de los horarios escolares y la necesidad de que éstos sean más flexibles para el curso siguiente. Además les gustaría que para el siguiente curso entraran más personas adultas en sus aulas. A partir de esta sesión algunos maestros se interesaron en ir a sus clases para ver cómo trabajaban y también para colaborar en las actividades que realizaban. En esta sesión se estableció el compromiso de que todas las personas entraran en alguna de las aulas de infantil o primer ciclo de primaria para participar en las sesiones u observar como se realizan los proyectos de trabajo, las tertulias dialógicas, los grupos interactivos y las lecturas inter-nivel. Este compromiso suponía una primera acción para las personas que participaban del taller de formación.

En la cuarta sesión, el profesorado que había entrado en las aulas de infantil o primaria compartió su experiencia. En la mayoría de los casos, los maestros y las maestras del centro entraron como observadores no participantes, para conocer el funcionamiento de los grupos interactivos y las tertulias dialógicas. Su entrada en la clase para observar y el compartir sus observaciones con las maestras que realizaron las prácticas supuso un encuentro más entre profesionales. El resultado de las observaciones realizadas desembocó en establecer pequeños compromisos en la sesión de formación. Fue una propuesta del equipo directivo que el profesorado acogió con entusiasmo. De manera que algunos maestros que en un principio habían manifestado su resistencia a implementar dichas prácticas acabaron realizando tertulias dialógicas, grupos interactivos y proyectos de trabajo.

En la quinta sesión se compartió la experiencia, de gran parte del profesorado al implementar cambios en sus prácticas. Fue una sesión en la que se discutieron muchos aspectos pedagógicos de la docencia. Al finalizar la sesión, se establecieron compromisos en cada uno de los ciclos para realizar prácticas inclusivas en el siguiente curso. En la etapa infantil se adquirió el compromiso de seguir realizando proyectos de trabajo; incorporar la actividad "El protagonista de la semana", fomentando la entrada de las familias en el aula; realizar tertulias dialógicas en cinco años e incorporar las lecturas inter-nivel, para que alumnos de ciclos superiores fueran a su aula a leerles un cuento. En primer ciclo de primaria, se comprometieron a mantener las tertulias dialógicas, la lectura inter-nivel y los grupos interactivos. Además de realizar proyectos de trabajo en la asignatura de conocimiento del medio. En el caso de la lectura inter-nivel, se comprometieron a realizarla también con otros 
ciclos. El segundo ciclo de primaria, se comprometieron a hacer algún proyecto de trabajo durante el curso; realizar grupos interactivos; tertulias dialógicas y lectura inter-ciclos. En el caso del tercer ciclo de primaria, como no se conocía al profesorado que impartiría esos cursos ya que todavía no formaba parte de la plantilla del centro, no se fijó ningún compromiso. Sin embargo, se insistió en un compromiso de centro por el que establecieron que las prácticas inclusivas irían promocionando a lo largo de los cursos junto con el alumnado. De manera que, una vez el alumnado del segundo ciclo pasara al tercer ciclo, los maestros deberían trabajar en el aula las prácticas inclusivas que se realizaban también en el ciclo anterior.

A continuación destacaremos los principales resultados fruto del seminario de formación. En concreto, realizamos una primera aproximación a los resultados que nos permiten responder por qué y cómo se han transformado las prácticas de aula. Además de conocer los cambios alcanzados en otros aspectos del centro.

\section{Resultados}

Aunque los resultados de la experiencia nos permiten entender por qué y cómo se han transformado las prácticas de aula, el hecho de que éstas se hayan transformado, en la mayoría de las clases, ya es un indicador del trabajo realizado en el seminario.

Sin embargo, entenderemos mejor a que se deben estos cambios a partir de los datos emergentes en las entrevistas, grupos de discusión, sesiones del seminario y en las prácticas realizadas en las aulas.

Por qué se transforman las prácticas de aula en esta escuela se debe a distintas causas. Para entenderlas haré referencia a las sesiones del seminario. A lo largo de las mismas, fuimos observando distintos posicionamientos entre el profesorado. Cada una de las sesiones realizadas trataba sobre aspectos pedagógicos de la acción del docente y cuestionaba las prácticas de maestros y maestras del seminario. Este hecho era del todo esperable puesto que en un conjunto de personas existe diversidad de opiniones y creencias. Por una parte había personas con una ideología menos inclusiva que encontraban impedimentos a las prácticas más abiertas y centradas en un papel activo del alumnado. Por el otro, había personas que defendían las prácticas inclusivas y las habían puesto en marcha, o mostraban su interés y motivación para hacerlo. Lo sorprendente fue que personas que no creían en las prácticas presentadas en el seminario las realizaron en ese mismo curso. Tras la observación de las prácticas que estas personas realizaron se dejaba 
entrever una distancia entre la práctica realizada y la teoría que sustentaba a la práctica. Es decir, había maestras que realizaron grupos interactivos, pero en ellos no se trabajó la autonomía del alumnado, ni la atención a la diversidad. En estos casos, en la entrevista que realizamos para conocer porqué habían realizado grupos interactivos, las maestras comentaron que "por probar", ya que se habían comprometido en realizar estas prácticas al curso siguiente. Una de las docentes que no encontraba sentido a estas prácticas comentó que las había realizado para "probarlas" y si no le gustaban al curso siguiente no realizarlas.

Por otra parte, también hubo docentes que mostraban más apertura a incorporar innovaciones en sus aulas. Éstos, a lo largo del curso fueron incorporando nuevas técnicas inclusivas. Las razones eran varias pero todas coincidían en querer fomentar una mayor autonomía en su alumnado, un aprendizaje más significativo y funcional para la vida. La observación de estas prácticas mostraba docentes flexibles que daban protagonismo al alumnado haciéndole responsables de su propio aprendizaje. Eran prácticas donde se fomentaba la toma de decisiones democrática, un aprendizaje dialógico y una apertura a la comunidad y al barrio. En una de las entrevistas, una maestra me comentó que la puesta en práctica de estas técnicas había ido modificando sus creencias y concepciones pedagógicas.

Sobre el cómo se fueron modificando las prácticas podemos encontrar su evolución en las sesiones del seminario y las observaciones de las prácticas en el aula. El seminario fue un contexto idóneo para aprender nuevas estrategias pero sobretodo, para replantearse cada uno su posicionamiento pedagógico. A lo largo de las sesiones se intentaba que las reflexiones realizadas en las mismas llegaran a acciones en el aula. Por eso, las prácticas se transformaron con el acompañamiento de todo el claustro y con el apoyo de otros profesionales que ya las habían puesto en práctica. La transformación pretendía realizarse de prácticas tradicionales a prácticas o acciones de éxito que fomentaban la inclusión del alumnado. Sin embargo, no siempre resultaba fácil propiciar dicha inclusión o un aprendizaje significativo entre el alumnado. Para algunas maestras que participaron en grupos interactivos como voluntarias, romper con un papel más directivo para pasar a ser gestoras de las interacciones del alumnado, era complicado. Por tanto, el cómo se transformaron las prácticas dependió en cada uno de los docentes, de sus creencias y del acompañamiento recibido en la realización de las prácticas. En general, podemos hablar de una transformación de prácticas con carácter tradicional a prácticas que tratan de fomentar la inclusión. Aunque no siempre los grupos interactivos realizados cumplían con su 
objetivo, ya que en algunos casos, la intención de las maestras, conscientes o no de ello, no era la inclusión, sino un modo diferente de agrupación y de dinámica en el aula.

Los cambios que se realizaron en el centro a partir de la realización del seminario fueron muy diversos. En primer lugar, todo el equipo de docentes empezó a conocer las prácticas que se estaban realizando en la escuela. Además, se empezó a reconocer y valorar el trabajo de compañeros que realizaban prácticas a favor de la escuela que intentaban construir. Reconocimiento que en la mayoría de los casos vino acompañado de la colaboración y acompañamiento entre los docentes para implementar cambios en el aula.

En segundo lugar, el posicionamiento del profesorado también cambió. Además del cambio en las prácticas, el profesorado era un investigador de su práctica y de la de sus compañeros. Desde el seminario se contagiaron experiencias inclusivas, se interpeló a compañeros a partir de la crítica constructiva y se responsabilizó al profesorado de los cambios que se consiguieron en el aula.

Además, a partir del seminario surgieron proyectos compartidos entre profesorado de diferentes ciclos y se reflexionó sobre la necesidad de que la escuela aprenda y de aunar objetivos que propicien un cambio real para conseguir responder a las necesidades de todos los miembros. En este sentido, el seminario de formación fue un punto de referencia para el profesorado del centro. Un espacio donde compartir prácticas para tratar de fomentar la inclusión desde las aulas.

Otra de las mejoras que favoreció el seminario fue la apertura a la comunidad. Las comunidades de aprendizaje implican un cambio de los hábitos de comportamiento habituales tanto para las familias como para el profesorado y el alumnado (Elboj y cool., 2002). Con la entrada de las familias en las aulas se rompió con la estructura rígida del contexto escolar y con la creencia de que quien enseña es el docente. En la experiencia que contaron las maestras de primer ciclo, se visibilizó a las familias como una parte importante de la comunidad educativa que favoreció también el aprendizaje del alumnado. Este aspecto implicó también un cambio en las bajas expectativas que se tenía de las familias y propició una mayor participación de éstas en el centro. Se fomento la idea que defienden Alcalde y cool. (2006) de que la escuela debe crear canales de relación entre el centro y las familias.

Además, el trabajo colaborativo, tanto entre profesores como entre familias, alumnado y profesorado dentro del aula, ha supuesto en sí un aprendizaje dialógico (Aubert, Flecha, García, Flecha, Racionero, 2008). En el caso del profesorado, la relación de igualdad era una realidad en las 
sesiones de formación. En el caso de las familias que entraban en las aulas, las maestras remarcaban muchas veces que todas las personas del aula eran iguales, y en todo momento se intentaba fomentar un diálogo igualitario.

\section{Discusión}

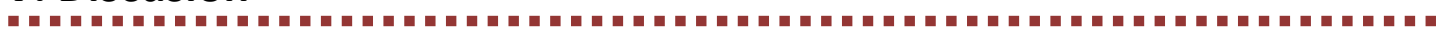

De los resultados presentados podemos destacar dos aspectos principalmente: las concepciones o creencias del profesorado a la hora de implementar cambios en su práctica y el acompañamiento y trabajo compartido entre el profesorado como un aspecto facilitador del cambio.

En el caso de las concepciones pedagógicas del profesorado remitimos a Blanco (1999) y Parrilla (2002) cuando hablan de la inclusión como una forma de vida. Es decir, para las maestras y maestros que entienden la inclusión como un derecho incuestionable y no conciben otra manera de educar, sus prácticas trataran de ser flexibles y adaptarse a las necesidades del alumnado. Por lo contrario, quienes no entienden la inclusión como una necesidad vital necesitaran de un esfuerzo para que al realizar acciones educativas de éxito, éstas incluyan a todo su alumnado y propicien valores de la inclusión. Por esta razón, en los resultados remarcamos que no todos los grupos interactivos realizados en la escuela conseguían los mismos resultados. No en todos los casos se propiciaba una mayor autonomía entre el alumnado ni el profesorado facilitaba la inclusión. Sin embargo, sí que es cierto que aumentaba el trabajo realizado por el alumnado. Por otra parte, en el caso que estudiamos, incluso la formación recibida por el profesorado no era suficiente para que los grupos interactivos se realizaran de manera adecuada. Como dicen Flecha y Puigvert (2002) en cada grupo interactivo, la persona voluntaria trata de dinamizar las interacciones entre el alumnado. Por el contrario, si no se fomenta dicha interacción entre iguales sino que la persona adulta es quien resuelve las dudas, estaremos limitando el aprendizaje.

Como dice Saiz, «intercambiar experiencias nos permite enseñar y aprender de los errores y aciertos de los demás. Es una necesidad que nos permite avanzar más rápidamente hacia la inclusión sin tener que empezar de cero continuamente» (2009: 23). Esta idea que justifica las interacciones entre iguales nos permite acercarnos a las interacciones entre el profesorado. Puesto que las sesiones de formación han sido un revulsivo en la puesta en práctica de acciones educativas de éxito.

Además, esta experiencia está promoviendo en el centro un mayor apoyo y asesoramiento colaborativo desde los recursos humanos del propio centro. Recibiendo la ayuda de los miembros de la comunidad y el 
asesoramiento de otras personas que entran a formar parte de nuestra experiencia.

La transformación de esta escuela en comunidad de aprendizaje todavía está iniciándose. Ésta puede ser una razón por la que encontramos contradicciones entre los cambios que se generan en el centro y el poco movimiento que hasta el momento parecía haber en las aulas. Sin embargo, siendo un centro que se inicia, ya se han encontrado muchos aspectos positivos que favorecen la inclusión. Se ha fomentado el trabajo colaborativo entre el profesorado, la innovación en el aula enfocada a generar prácticas inclusivas, utilizar metodologías variadas y buscar soluciones ante las dificultades (Equipo de maestros del CEIP Folch i Torres, 2009).

Agradecimientos: equipo de docentes, conserje, familias y alumnado del CEIP Francesc Roca i Alcaide.

\section{Bibliografía}

Alcalde, A.; Buitago, M.; Castanys, M.; FálCes, M.P.; Flecha, R.; González, P.; Jaussi; Ma L. Li Lavado, J.; Odina, M.; Ortega, S.; Palenzuela, A.; Planes, LI.; Puigdellívol, I.; RAMIS, M.; RuBio, A.; WellS, G. (2006). Transformando la escuela: las comunidades de aprendizaje, Graó, Barcelona.

Aubert, A., Flecha, A., García, C., Flecha, R., Racionero S. (2008). Aprendizaje dialógico en la sociedad de la información, Hipatia Editorial, Barcelona.

Blanco, R. (1999). "Hacia una escuela para todos y con todos». Boletín del Proyecto principal de educación en América Latina y el Caribe, 48, pp.55 72.

Bolívar, A. (1996). "Cultura escolar y cambio curricular». Bordón, 48 (2), pp. 169-177.

Bоoth, T. (2006). "Manteniendo el futuro con vida; convirtiendo los valores de la inclusión en acciones» en VERDUGO, M.A. y JORDÁN DE URRíES, F.B. (eds.): Rompiendo inercias. Claves para avanzar. VI Jornadas Científicas de Investigación sobre Personas con Discapacidad, Amarú, Salamanca.

Escobedo, P. (2010). La cultura de un centro en su camino hacia la inclusión. (Tesis inédita de maestría). Universitat Jaume I, Castellón.

EquiPo de MAESTROS DEL CEIP FolCH I TORRES (2009). "La inclusión en el CEIP Folch i Torres de Esplugues de Llobregat: un modelo institucional» en Macarulla, I. y SAIZ,M. (eds.): Buenas prácticas de escuela inclusiva. La 
inclusión de alumnado con discapacidad: un reto, una necesidad (pp. 85111), Graó, Barcelona.

EssombA, M.A. (2006): Liderar escuelas interculturales e inclusivas. Equipos directivos y profesorado ante la diversidad cultural y la inmigración, Graó, Barcelona.

FlechA, R.; PUIGVert, L. (2002): "Las comunidades de aprendizaje: una apuesta por la igualdad educativa». REXE Revista de estudios y experiencias en educación, 1, (1), pp. 11-21. Consultado el 9 de septiembre del 2010 en http://www.uscs.cl/rexe

Grañeras, M., DíAZ, P. y GIL, N. (2011). "Actuaciones de éxito en las escuelas europeas». Colección Estudios CREADE, 9. Ministerio de Educación.

GómeZ, J. (2004). "Metodología comunicativo crítica» en BISQUERRA, R. (ed.): Metodología de la investigación educativa (pp. 395 - 424), La Muralla, Madrid.

LATORRE, A. (2004). "La investigación - acción» en BISQUERRA, R. (ed.): Metodología de la investigación educativa (pp. 369- 394). La Muralla, Madrid.

LEWIN, K. (1946) "Action research and minority problems», Journal of Social Issues, 2, pp. $34-46$.

ORTIZ, MaC. Y LOBATO, X. (2003). "Escuela inclusiva y cultura escolar: algunas evidencias empíricas», Bordón, 55, (1), pp. 27- 40. Consultado el 6 de junio del 2010 en http://www.pasoapaso.com.ve/CMS/images/stories/Integracion/gemas 205.pdf

Parrilla, A. (2002). "Acerca del origen y sentido de la educación inclusiva», Revista de Educación, 327, pp. 11- 29.

SAIZ, M. (2009). "El modelo educativo que queremos» en MACARULLA, I. y SAIZ,M. (eds.): Buenas prácticas de escuela inclusiva. La inclusión de alumnado con discapacidad: un reto, una necesidad (pp. 9 - 33), Graó, Barcelona. 
